\title{
Proceedings of the 11th Congress of the International Society of Nutrigenetics and Nutrigenomics (ISNN 2017)
}

\author{
William T. Barrington ${ }^{a}$ Anna C. Salvador ${ }^{b}$ Jaana A. Hartiala ${ }^{c}$ \\ Raffaele De Caterina ${ }^{d}$ Martin Kohlmeier ${ }^{e} f \quad$ J. Alfredo Martinez ${ }^{g-j}$ \\ Carin B. Kreutzerk ${ }^{k}$ David Heber' Aldons J. Lusisa ${ }^{a}$ Zhaoping Li' \\ Hooman Allayee ${ }^{c}$ \\ a Departments of Human Genetics and Medicine, David Geffen School of Medicine of UCLA, \\ Los Angeles, CA, ${ }^{\mathrm{b}}$ Departments of Nutrition and Food Science, and Molecular and Cellular \\ Medicine, Texas A\&M Health Science Center, College Station, TX, and ' ${ }^{\mathrm{C}}$ Departments of \\ Preventive Medicine and Biochemistry \& Molecular Medicine, Keck School of Medicine,

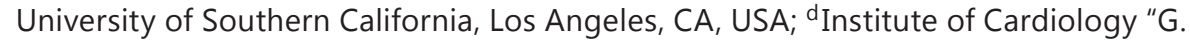 \\ d'Annunzio" University and Center of Excellence on Aging, Chieti, Italy; ${ }^{e}$ Department \\ of Nutrition, Schools of Medicine and Public Health, University of North Carolina at \\ Chapel Hill, Chapel Hill, NC, and fUNC Nutrition Research Institute, Kannapolis, NC, USA; \\ gDepartment of Nutrition, Food Science, and Physiology and Center for Nutrition Research, \\ University of Navarra, and h Navarra Institute for Health Research (IdiSNA), Pamplona, \\ iCIBERobn, Physiopathology of Obesity, Carlos III Institute, and ${ }^{\mathrm{j}}$ Madrid Institute of \\ Advanced Studies (IMDEA Food), Madrid, Spain; kLeonard Davis School of Gerontology, \\ University of Southern California, and 'Division of Clinical Nutrition, Department of \\ Medicine, and Center for Human Nutrition, David Geffen School of Medicine of UCLA, \\ Los Angeles, CA, USA
}

\section{Keywords}

Nutrigenetics · Nutrigenomics · Health · Disease $\cdot$ Gene-dietary interactions

\begin{abstract}
The International Society of Nutrigenetics and Nutrigenomics (ISNN) held its 11th annual Congress in Los Angeles, California, between September 16 and 19, 2017. In addition to 2 keynote lectures, 4 plenary sessions included presentations by internationally renowned speakers on cutting-edge areas of research and new discoveries in genetics/genomics, the microbiome, and nutrition. Scientific topics included multi-omics approaches; diet and the microbiome; cancer, longevity, and metabolism; moving the field forward; and translational/educational
\end{abstract}

William T. Barrington, Anna C. Salvador, Zhaoping Li, and Hooman Allayee contibuted equally to this work.

Hooman Allayee, PhD

Departments of Preventive Medicine and Biochemistry \&

Molecular Medicine, Keck School of Medicine of USC

Los Angeles, CA 90033 (USA)

E-Mail hallayee@usc.edu
Zhaoping Li, MD, PhD

Department of Medicine and Center for Human Nutrition David Geffen School of Medicine of UCLA

Los Angeles, CA 90095 (USA)

E-Mail zli@mednet.ucla.edu 
Barrington et al.: Proceedings of the 11th Congress of the International Society of Nutrigenetics and Nutrigenomics (ISNN 2017)

aspects and the future of medicine. There was also an accepted oral abstracts session designed specifically to provide young investigators and trainees with the opportunity to present their work, as well as a session focused on industry-academic partnerships, which included a roundtable discussion afterwards. Overall, the 11th ISNN Congress was an exciting and intellectually stimulating meeting focused on understanding the impact of biological interactions between genes and nutrients on health and disease. These efforts continued the decade-long tradition of the annual ISNN Congress to provide an interdisciplinary platform for scientists from various disciplines to discuss research ideas and advance the fields of nutrigenetics and nutrigenomics.

(c) 2018 S. Karger AG, Basel

\section{Introduction}

Since its inception in 2005, the International Society of Nutrigenetics and Nutrigenomics (ISNN) has held an annual international Congress to gather leading experts in the fields of genetics and nutrition in a collaborative environment and to foster interactions and the exchange of ideas on timely topics in nutrigenetics and nutrigenomics. Past Congresses have been held all over the world, including Europe, Brazil, China, the Middle East, Australia, and North America. This year, the 11th Congress of the ISNN (ISNN 2017) was held between September 16 and 19,2017, at the Meyer and Renee Luskin Conference Center on the campus of the University of Los Angeles, California. More than 200 attendees from 21 countries enjoyed fascinating lectures in the warm, sunny climate for which southern California is known. Dr. Hooman Allayee, Professor of Preventive Medicine and Biochemistry and Molecular Medicine at the University of Southern California, and Dr. Zhaoping Li, the Lynda and Stewart Resnick Endowed Chair in Human Nutrition and Director of the Center for Human Nutrition at the University of California, Los Angeles (UCLA), organized ISNN 2017 with the help of local organizers Drs. Jaana Hartiala (USC), Aldons J. Lusis (UCLA), Carin B. Kreutzer (USC), and David Heber (UCLA). During the plenary sessions, researchers, clinicians, and dietitians presented cutting-edge science and discussed strategies for improving dietary recommendations by accounting for individual differences with respect to genetics, epigenetics, sex, and gut microbiome composition. A training session provided guidance on how to best utilize the current understanding of nutrigenetics to improve outcomes in practice. Platform talks and poster presentations from young investigators afforded budding scientists the opportunity to share and discuss their research. The scientific program is provided in the online supplementary material (see www.karger.com/doi/10.1159/000485799 for all online suppl. material and available online at https://isnn2017.org/program/), and abstracts that were accepted for oral or poster presentation were recently published [1].

\section{ISNN Training Course "Nutrigenetics in Practice" - September 16, 2017}

Prior to the official opening of ISNN 2017, a training workshop was held, which served as a primer to the use of nutrigenetics in various healthcare fields. The workshop included a glossary of terms that would be used during the conference, as well as guidance on reading genomic information. One of the primary goals of the training course was to educate attendees on the basics of implementing nutrigenetics into clinical practice. The overall concept emphasized was that nutrigenetics aims to predict responses to diet through a better understanding of the individual's genetic background, and that dietary intake guidance may vary for a patient who is a responder versus a non-responder to a dietary intervention. 
Barrington et al.: Proceedings of the 11th Congress of the International Society of Nutrigenetics and Nutrigenomics (ISNN 2017)

Dr. Martin Kohlmeier and Olivia Dong shared several case studies to describe scenarios in which individuals harboring common genetic variants are more likely to benefit from genotype-directed guidance than from one-size-fits-all dietary recommendations. They illustrated the ethical and legal implications that would accompany this potential shift in clinical practice. Cancer [2-5] and cardiovascular health [6] were addressed with regard to genotype by diet interactions, as well as several nutrient-specific discussions on lactose intolerance [7, 8] and folate [5] requirements. As more genetic polymorphisms are considered for practice decisions, nutrigenetics was thought to improve precision of dietary recommendations on an individual level.

\section{Special Symposium “Health Benefits of Prebiotics” - September 16, 2017}

After the ISNN training session, all attendees were invited to a special symposium covering the health benefits of prebiotics. Dr. David Heber introduced prebiotics and discussed improved balance of gut microbiota experienced by feeding and fostering the microbiome with prebiotics. This was complemented by Dr. Jennifer Gu's talk on the modulating effects of xylo-oligosaccharides on individual flora and the improvement in intestinal function experienced by individuals suffering from irritable bowel syndrome.

\section{Keynote Lectures}

ISNN 2017 opened on September 17, 2017 (see online suppl. material) with the first of two keynote lectures delivered by Dr. David Heber, Professor Emeritus in the Department of Medicine and past Director of the Center for Human Nutrition at UCLA. Dr. Heber began with a historical context for the growing appreciation of individual differences and ended with a promising view for the future of nutrigenetics.

Dr. Heber described early philosophers and scientists, who understood that each person has unique idiosyncrasies that are important for the constitution of the individual. Advancing a few millennia, Dr. Heber provided an overview of the scientific literature that demonstrates the importance of genetic differences in response to diet. He discussed the importance of evolutionary considerations when evaluating diet and stated that "We are well adapted to starvation, but poorly adapted to overnutrition." To illustrate this point, he used the example that, compared to Europeans, Native American and other indigenous populations are resistant to starvation but susceptible to diseases of overnutrition $[9,10]$. Dr. Heber provided specific examples in which subsets of individuals in a population can benefit from dietary modifications. Dr. Heber discussed his research on the role of polyphenols as prebiotics, and the varying impact of the metabolite, urolithin, on health status across people with different "metabotypes," or distinct metabolic types. Dr. Heber concluded with an optimistic view on how this growing body of knowledge can be used to improve health at an individual level.

Prior to the Plenary Sessions on day 2 of ISNN 2017, September 18, 2017, the second keynote lecture was delivered by Dr. Rob Knight, the Director of the Center for Microbiome Innovation and Professor of Pediatrics and Computer Science and Engineering at the University of California, San Diego. Dr. Knight described the significance of the gut microbiome for a wide range of health parameters and ended with a creative and innovative view of the future of health monitoring.

Dr. Knight began by comparing our complexity as a human to the complexity of the microorganisms we host. The complexity of the microbial metagenome is far greater than that of humans, with $\sim 2-20$ million genes compared to $\sim 20,000$ human genes, underscoring the 
Barrington et al.: Proceedings of the 11th Congress of the International Society of Nutrigenetics and Nutrigenomics (ISNN 2017)

challenge of identifying microbial gene functions and determining their impact on human health. Despite this complexity, Dr. Knight described the power of the microbiome to inform health status, noting that analysis of gut bacterial composition could predict obesity better than known genetic factors from human genome-wide association studies. Dr. Knight discussed studies in monozygotic twins that have shaped our understanding of the importance of the gut microbiome in individuals with identical genetics, and the use of microbiota transfers from humans into rodent models that validate the associations observed in people. Dr. Knight discussed findings from the American Gut Project, a crowdsourced, citizen science investigation that he initiated to explore gut microbial differences between people. He acknowledged that huge amounts of data have been collected from microbiome projects, and the challenge now is to transform this mass of data into actionable information. Dr. Knight ended his lecture with an exciting, imaginative view about the future of microbiome research and healthcare. He envisioned a "microbial GPS," which could predict a person's long-term health outcomes given their current gut microbial composition, and provide personalized recommendations to alter the gut microbiome in a favorable direction.

\section{Plenary Sessions}

Multi-Omics Approaches to Nutrigenetics - September 17, 2017

The first Plenary Session of ISNN 2017 opened with state-of-the art presentations demonstrating how integrating multiple types of "omics" data, such as genomics, transcriptomics, epigenomics, metabolomics, lipidomics, etc., is key for fully elucidating the impact of diet (see online suppl. material). After Dr. Heber's keynote lecture, Dr. A. Jake Lusis opened the multiomics plenary session with a discussion of studies from his laboratory using a large panel of inbred mouse strains (Hybrid Mouse Diversity Panel) to model the diverse health effects of dietary perturbations. Dr. Lusis described how a wide range of health parameters are impacted differently by diet depending on genetics, and described novel methods to identify the genetic architecture underlying diet response. Next, Dr. David James described his lab's use of a large, genetically diverse panel of Drosophila strains (Drosophila Genetic Reference Panel) to investigate obesity-related genes in the context of specific dietary modifications. His results led to a conclusion that was echoed by several researchers during the conference, "There is a healthy diet optimum that differs by strain." Dr. Jimmy Bell discussed the practical use of genetics in clinical settings, noting that statistical changes are not always clinically relevant, and that the potential impact must be weighed against costs when determining whether to genotype patients. Dr. Martinez discussed the use of genetic risk scores on obesity prediction, and demonstrated that the macronutrient ratio of a diet interacts with genetic risk scores to modify the association with adiposity. Then, Dr. Xu Lin shared results from large-scale studies in China that examined dietary impacts on metabolic syndrome and the role of folic acid in preeclampsia. The session concluded with Dr. Allayee's demonstration of how genetics can be used to establish whether a given biomarker is causative or reactive. He provided informative examples from his studies on betaine and its association with cardiovascular disease risk [11].

\section{Longevity, Metabolism, and Cancer - September 17, 2017}

The central theme of the second Plenary Session was how interindividual response to diet can have serious implications for longevity, metabolism, and cancer (see online suppl. material). Dr. Pinchas Cohen began by describing regulation by mitochondrial-derived peptides on diet response and effects on longevity. Dr. Brian Bennett then touched on the necessity of translating discovery science into formal dietary recommendations. He noted the strong association between dietary adherence and efficacy in diet studies and questioned 
Barrington et al.: Proceedings of the 11th Congress of the International Society of Nutrigenetics and Nutrigenomics (ISNN 2017)

which factor was the driving force. He then discussed his research of microRNAs in dietary response in genetically distinct mice. Dr. William Evans recounted his work developing new methods for the evaluation of skeletal muscle integrity as a means to identify the presence of sarcopenia in adults and how this has led to recategorization of clinically relevant muscle wasting. Dr. Mariana Stern shared her work regarding the relationships between polymorphisms of carcinogen metabolism genes, red meat consumption, and cancer risk. Dr. Charles Brenner continued to discuss the conversion of discovery science into formal recommendations while speaking about nicotinamide riboside as a precursor to NAD+ and health benefits to maintaining adequate NAD+ levels. Dr. Joseph Pisegna wrapped up the session with a discussion on the use of high-protein diets for amelioration of metabolic disease and the potential mechanisms underlying the effects. This concluded the first day of the 11 th Congress of the ISNN.

Diet and the Microbiome - September 18, 2017

The third Plenary Session on day 2 of ISNN 2017 began with multifaceted presentations on the importance of the microbiome in nutrigenetics/nutrigenomics (see online suppl. material). Over the last decade, the role of the gut microbiome in human health has become among the most promising scientific fields. Given the growing appreciation for the gut microbiome's impact on a variety of diseases, including metabolic diseases, and the ability of diet to alter gut microbiome composition, the "Diet and the Microbiome" Plenary Session was eagerly anticipated. After the keynote lecture by Dr. Knight, Dr. Stanley Hazen shared the results of his studies uncovering the mechanisms by which the gut bacteria-derived metabolite, trimethylamine $\mathrm{N}$-oxide, promotes atherosclerosis under certain dietary conditions [12]. Next, Dr. Jonathon Braun presented on the role of the microbiome in inflammatory bowel disease (IBD). His work identified microbial and metabolomic features that can be observed before the onset of IBD, suggesting that microbiota play a causal role in disease susceptibility. Dr. Sean Adams shared his work on the use of resistant starch to ameliorate various models of disease in mice without altering other health parameters. Next, Dr. Elaine Hsiao presented her work on the role of gut microbiota and the utility of a ketogenic diet for ameliorating seizure activity in individuals with epilepsy. Using mouse models, she identified that specific combination gut bacteria that can produce similar benefits without following a restrictive diet. Finally, Dr. Li concluded the session by discussing the current challenges of determining how diet influences the microbiome and the opportunities to improve health through modification of the gut microbiome.

Industry/Academic Partnerships for Moving the Field Forward - September 18, 2017

The fourth Plenary Session included several speakers who provided insight into how industry and academic partnerships can move the fields of nutrigenetics and nutrigenomics to public access points (see online suppl. material). Dr. Christopher Lynch launched the session by describing the task force formed by the NIH to develop the first strategic plan for $\mathrm{NIH}$ nutrition research across all domains of nutrition-related diseases and conditions. This effort aims to enhance and unite ongoing and future research in the field. Dr. John Courtney brought yet another perspective, speaking from a chief executive officer's point of view, on efforts to work across the public-private sectors to identify approaches to improve public trust in nutrition science. Dr. Orian Shirihai described nutrient effects on mitochondrial quality control observed during his work investigating the mitochondrial life cycle and efficiency. Dr. Ari Mayer Mackler delivered comments regarding proper industry funding of research through transparency of principles, responsibility, and product alignment to company mission statements. Dr. Ahmed El-Sohemy spoke in favor of genetic testing companies that have already begun to implement nutrigenetics and nutrigenomics in practice, 
Barrington et al.: Proceedings of the 11th Congress of the International Society of Nutrigenetics and Nutrigenomics (ISNN 2017)

citing key studies on metabolic modifier genes involved in gene by environment interactions underlying single SNPs and metabolic response. The final speaker of the day was Dr. Ray Jiao, who shared his work on an early warning system for cancer screening and targeted nutrition.

Following the talks, a round table session was held to discuss current issues around nutrigenetics and nutrigenomics. Dr. Martin Kohlmeier led the panel discussion with Drs. Lynch, Shirihai, Mayer, Mackler, El-Sohemy, and Jiao, who were joined by Dr. Changhao Sun, who is Vice President of Harbin Medical University and Professor and Chairman of the Department of Nutrition and Food Hygiene in Harbin, China. Topics of the discussion included private versus public support of science; variation in funding structures between nations; how to increase transparency of science and promote public trust in science; and how to monitor and improve the reproducibility of studies. Predictably, the importance of genetic considerations in nutritional studies was a major topic of discussion. When discussing his appreciation for the importance of nutrigenetics in nutrition studies, Dr. Mayer Mackler described the need to transverse an absence of knowledge in certain areas in order to provide something meaningful for customers, and the role of private funding to advance our understanding of nutrigenetics. Dr. El-Sohemy asserted that given our knowledge about the importance of genetic considerations in evaluating diet response, genetics should now be an essential component of every nutrition study.

The panel also discussed the important need and role of research funding for nutrition studies. Panelists commented on the potential for bias or perceived bias of nutrition studies funded by private companies to investigate products that the company sells. The panel described the need for private funding to further our understanding of nutrition, with Dr. Mayer Mackler noting that private funding can be used for pilot studies to apply for larger grants from public organizations. Dr. El-Sohemy mentioned the need to "stick to the science" when reviewing studies, rather than perceiving an inherent bias based on the funding source. Dr. Sun provided an international perspective, noting that the government largely funds nutrition studies in China with a comparatively small input from private businesses.

The discussion transitioned into methods for improving public perception of science research and the need for transparency. Dr. Shirihai expressed concerns about the public's access to research articles and their ability to make sense of scientific jargon. He suggested that perhaps a database could describe study results in lay language, although the panel voiced concerns about who would oversee such a database and how the studies would be interpreted.

The replication of study results was a central concern among the panel members. A key issue seemed to be that few studies are ever repeated, and those that are repeated often fail to replicate the original results. Dr. Lynch acknowledged that just because a result does not replicate in a follow-up study does not necessarily mean that someone was wrong as small environmental effects can impact outcomes. Members of the panel expressed concern about the difficulty of obtaining funding to replicate previous findings, to which Dr. Lynch mentioned that few such grant proposals are submitted to the NIH for consideration of funding. Dr. Shirihai suggested that perhaps government funding agencies could call for grants specifically for the replication of previous high-impact studies.

The round table concluded with an acknowledgement of the great impact that nutrigenetics and nutrigenomics can have on human health and the need to advance the field as quickly as possible while maintaining the integrity of results and cultivating the public's trust in science. This concluded the second day of the 11th Congress of the ISNN.

Accepted Oral Presentations and Young Investigator Talks - September 19, 2017

An important goal of the ISNN and its annual scientific meetings is to provide opportunities for young scientists to share their research with the nutrigenetics community. As such, 
Barrington et al.: Proceedings of the 11th Congress of the International Society of Nutrigenetics and Nutrigenomics (ISNN 2017)

the ISNN 2017 organizers encouraged the submission of abstracts for consideration of oral or poster presentations. Two poster sessions were held on September 17 and 18, 2017 [1], and the ISNN 2017 local organizing committee awarded speaking opportunities to several young scientists (see online suppl. material). Dr. William Barrington from UCLA began the session on Accepted Oral Presentations and Young Investigator Talks by presenting his work with genetically distinct mice, which demonstrated the profound influence of genetics on cardiometabolic health effects of several popular human diets. Dr. Jie Li from Harbin Medical University then discussed his findings on how prenatal exposure to famine affects the risk of type 2 diabetes in offspring. Dr. Tatiane Fujii from the University of São Paulo shared her work on how inflammation in a Brazilian population was influenced by interactions between polymorphisms in the fat mass and obesity-associated protein (FTO) gene and plasma levels of omega-3 fatty acids. Next, Dr. Jieping Yang from UCLA shared her results on how prebiotic fiber xylo-oligosaccharides alter gut microbiota and improve kidney function in mouse models of nephropathy. The session was concluded with Dr. Vimal Karani from the University of Reading in the UK who shared results from his genetic and epidemiological studies suggesting a causal link between milk consumption and cardiometabolic outcomes.

Translational/Educational Aspects and the Future of Medicine - September 19, 2017

To promote the maturation of nutritional science, the last Plenary Session of ISNN 2017 highlighted efforts that are underway to translate discovery science into educational resources for precision dietetics as a component of precision medicine (see online suppl. material). As the first speaker, Dr. Ronald Krauss' presentation paralleled nutrigenetics and nutrigenomics with his extensive experience in the pharmacogenetics and pharmacogenomics of LDL cholesterol lowering and determining diet by drug interactions. Dr. Goran shared evidence that fructose and glucose have separate pathways and fates with regard to liver dysfunction in Hispanic youth who are obese, which may have implications for therapeutic strategies as a function of genetic risk. Dr. Karin Michels discussed her studies on epigenetic status and identified the microbiome as a major regulator of dietary health effects. Dr. Kurt Hong followed this by introducing the concept that certain populations could benefit from diet modification using fasting or pseudo-fasting diets to promote health and longevity. Dr. Martin Kohlmeier announced that ISNN is currently developing guidelines for genotype-specific nutrition targets to achieve utility in practice for robust genetic markers with clear effects. Dr. Raffaele De Caterina followed up with plans for the production of an ISNN textbook, entitled "Principles of Nutrigenetics and Nutrigenomics." Finally, Dr. Vijaya Surampudi closed the last session of ISNN 2017 with a long-term vision of large-scale healthcare implementation of personalized nutrition.

\section{Looking Forward}

The fields of nutrigenetics and nutrigenomics hold great promise with much yet to be discovered. The current body of knowledge demonstrates that the health effects of diet are heavily influenced by genetics, epigenetics, and the gut microbiome. It is apparent that nutrition and diet recommendations must shift from a one-size-fits-all approach to an individualized approach. Yet, we have only begun to uncover actionable means to do so. The future of nutrigenetics and nutrigenomics is thus dependent on our ability to dissect the mechanisms underlying individualized dietary response, and develop methods that allow for precise, individualized nutritional strategies. Achieving these goals holds the promise of a brighter, healthier future. 
Nournal of
and
Nutrigenetics
Nutrigemics

\section{Acknowledgements}

The ISNN and the local organizing committee extend their sincere gratitude to the invited speakers, faculty members, student/trainees, clinicians, nutritionists/dietitians, and all other attendees for their participation in ISNN 2017. The ISNN also wishes to acknowledge the continued support and efforts of its executive officers, Hooman Allayee (President; USA), Martin Kohlmeier (President-Elect and Secretary/Treasurer; USA), J. Alfredo Martínez (Immediate Past-President and Board Member; Spain), and Raffaele De Caterina (Past-President and Board Member; Italy), as well as its Board Members, Agata Chmurzynska (Poland), Myung-Sook Choi (South Korea), Rui Curi (Brazil), Lynnette R. Ferguson (New Zealand), Jing X. Kang (USA), Ron Martin (USA), Louis Pérusse (Canada), Chandan Prasad (USA), and Artemis P. Simopoulos (ISNN Founder and Past-President; USA). Lastly, the ISNN and the local organizing committee would like to acknowledge and is especially grateful to the exhibitors and sponsors for their generous support of the 11th Congress of the ISNN: LONGLIVE (China), Nutrasumma (USA), The Wonderful Company (USA), UCLA Center for Human Nutrition (USA), USC Diabetes and Obesity Research Institute (USA), USC Department of Preventive Medicine (USA), USC Leonard Davis School of Gerontology (USA), L-Nutra (USA), Nutrigenomix (CAN), Chinese Nutrition Society (China), National Board of Physician Nutrition Specialists (USA), and the American Society of Nutrition (USA).

\section{References}

1 11th Congress of the International Society of Nutrigenetics/Nutrigenomics (ISNN): Abstracts. J Nutrigenet Nutrigenomics 2017;10:93-125.

2 Hedelin M, Balter KA, Chang ET, Bellocco R, Klint A, Johansson JE, Wiklund F, Thellenberg-Karlsson C, Adami HO, Gronberg H: Dietary intake of phytoestrogens, estrogen receptor-beta polymorphisms and the risk of prostate cancer. Prostate 2006;66:1512-1520.

3 Hedelin M, Chang ET, Wiklund F, Bellocco R, Klint A, Adolfsson J, Shahedi K, Xu J, Adami HO, Gronberg H, Balter KA: Association of frequent consumption of fatty fish with prostate cancer risk is modified by COX-2 polymorphism. Int J Cancer 2007;120:398-405.

4 Goodman M, Bostick RM, Ward KC, Terry PD, van Gils CH, Taylor JA, Mandel JS: Lycopene intake and prostate cancer risk: effect modification by plasma antioxidants and the XRCC1 genotype. Nutr Cancer 2006;55:13-20.

5 Xu X, Gammon MD, Wetmur JG, Rao M, Gaudet MM, Teitelbaum SL, Britton JA, Neugut AI, Santella RM, Chen J: A functional 19-base pair deletion polymorphism of dihydrofolate reductase (DHFR) and risk of breast cancer in multivitamin users. Am J Clin Nutr 2007;85:1098-1102.

6 Cornelis MC, El-Sohemy A, Kabagambe EK, Campos H: Coffee, CYP1A2 genotype, and risk of myocardial infarction. JAMA 2006;295:1135-1141.

7 Labrie V, Buske OJ, Oh E, Jeremian R, Ptak C, Gasiunas G, Maleckas A, Petereit R, Zvirbliene A, Adamonis K, Kriukiene E, Koncevicius K, Gordevicius J, Nair A, Zhang A, Ebrahimi S, Oh G, Siksnys V, Kupcinskas L, Brudno M, Petronis A: Lactase nonpersistence is directed by DNA-variation-dependent epigenetic aging. Nat Struct Mol Biol 2016;23:566-573.

8 Oh E, Jeremian R, Oh G, Groot D, Susic M, Lee K, Foy K, Laird PW, Petronis A, Labrie V: Transcriptional heterogeneity in the lactase gene within cell-type is linked to the epigenome. Sci Rep 2017;7:41843.

9 O'Dea K: Diabetes in Australian aborigines: impact of the western diet and life style. J Intern Med 1992;232: 103-117.

10 Schulz LO, Bennett PH, Ravussin E, Kidd JR, Kidd KK, Esparza J, Valencia ME: Effects of traditional and western environments on prevalence of type 2 diabetes in Pima Indians in Mexico and the US. Diabetes Care 2006;29: 1866-1871.

11 Hartiala JA, Tang WH, Wang Z, Crow AL, Stewart AF, Roberts R, McPherson R, Erdmann J, Willenborg C, Hazen SL, Allayee H: Genome-wide association study and targeted metabolomics identifies sex-specific association of CPS1 with coronary artery disease. Nat Commun 2016; 7:10558.

12 Koeth RA, Levison BS, Culley MK, Buffa JA, Wang Z, Gregory JC, Org E, Wu Y, Li L, Smith JD, Tang WH, DiDonato JA, Lusis AJ, Hazen SL: $\gamma$-Butyrobetaine is a proatherogenic intermediate in gut microbial metabolism of L-carnitine to TMAO. Cell Metab 2014;20:799-812. 